\title{
III. A method for comparing the values of the specific inductive capacity of a substance under slowly and rapidly changing fields: Results for paraffin and glass
}

\section{Edwin F. Northrup}

To cite this article: Edwin F. Northrup (1895) III. A method for comparing the values of the specific inductive capacity of a substance under slowly and rapidly changing fields: Results for paraffin and glass, Philosophical Magazine Series 5, 39:236, 78-92, DOI: $10.1080 / 14786449508620682$

To link to this article: http://dx.doi.org/10.1080/14786449508620682

$$
\text { 曲 Published online: } 08 \text { May } 2009 .
$$

Submit your article to this journal ¿

\section{щll Article views: 3}

Q View related articles ¿ 


\section{$\left[\begin{array}{ll}78 & \end{array}\right]$}

III. A Method for Comparing the Values of the Specific Inductive Capacity of a Substance under Slowly and Rapidly Changing Fields: Results for Paraffin and Glass. By EDWIN F. NorThruP*.

[Plate 1II.]

$\mathbf{M}^{\prime}$ AXWELL'S electromagnetic theory of light entails the relation that the square of the index of refraction of a substance, for infinitely long waves, shall equal its specific inductive capacity. But as the refractive index has only been obtained for very short waves (excepting some experiments upon the refractive index of a few dielectrics for electric waves), it is not unexpected that this relation should not hold in the case of many substances whose specific inductive capacity has been determined in steady or very slowly alternating fields. It is to be expected, however, if the field of force under which the value of $\mathrm{K}$ is obtained could be made to alternate at a rate which would produce waves comparable in length to the waves with which the-refractive index is determined, that the agreement of $K$ and $\mu^{2}$ required by the theory would be obtained. The experiments of Professor Hertz upon electrical oscillation have suggested certain methods for determining the specific inductive capacity under fields changing at a rate which approaches that required. No research, however, aiming at a direct comparison of the values of $\mathrm{K}$ under slowly changing and oscillating fields, which is as fully conclusive as could be desired appears to have been made. The objects held in view in the following investigation were :-1st. To perfect a method which will enable the specific inductive capacity of solids (for which substances the value of $\mathrm{K}$ departs most from the required relation) to be determined under fields of force varying slowly or rapidly as desired ; and 2nd. To employ the method on one or two substances, to learn if the agreement with the theory is closer in the latter than it is in the former case. With the method under consideration this comparison may be made with the same apparatus and under conditions similar in all essential features.

\section{Description of Apparatus.}

Fig. 1, Pl. III., gives in elevation the apparatus employed. Fig. 2 in plan. The dimensions of the parts are given in the diagram in centimetres. The following are its essential

* Communicated by the Author. 


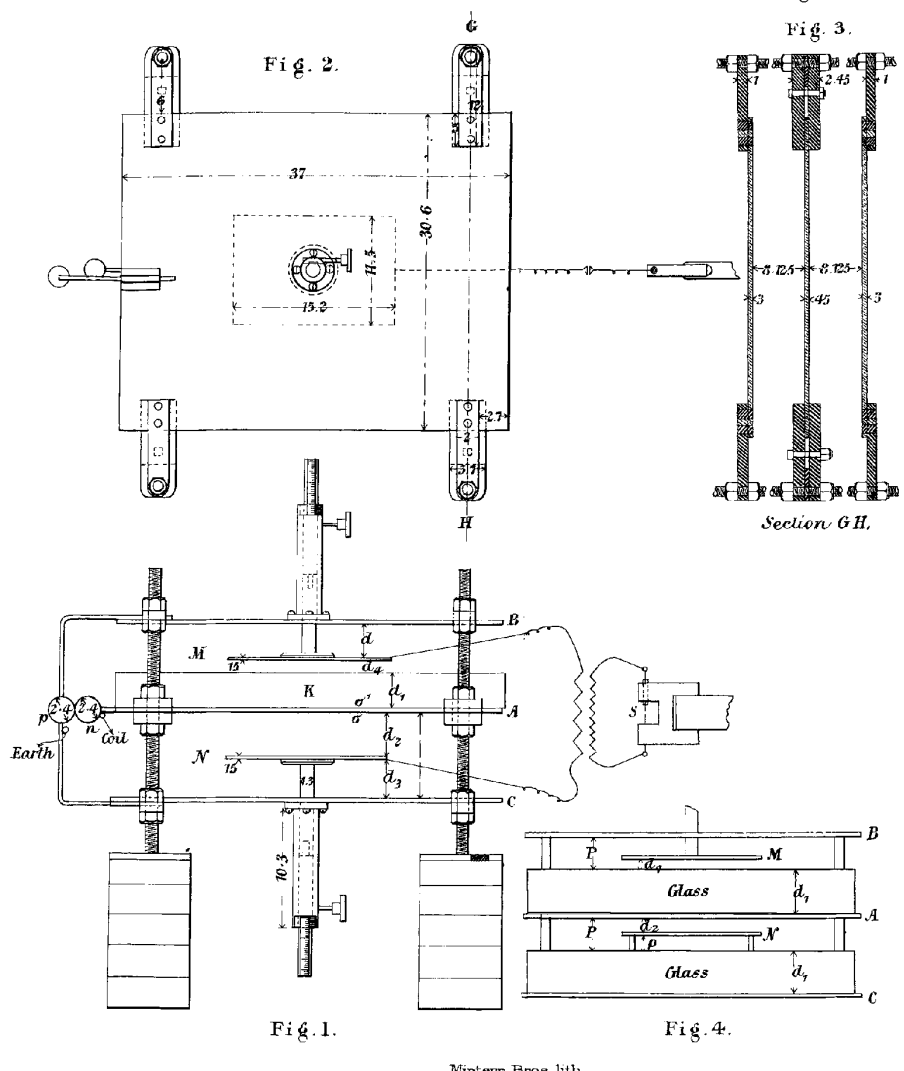


features :-Three heavy brass plates, A, B, and C (fig. 1), each $12 \times 14$ inches, are fastened parallel to one another, and are capable of being separated each from the other through a range of several inches. Each plate is held in position by four insulating strips of ebonite which extend out from its edge ; four iron rods, with a screw-thread cut upon their entire length, pass through the ends of the ebonite strips, which may be secured in any position upon the rods by bolts one above and one below each strip (figs. 1, 2, and 3). At the centre of each of the two outside plates, and perpendicular to them, is fastened a heavy brass tube. A rod, half of which is brass and half ebonite, slides in this tube, the ebonite portion extending between the plates; and the whole is capable of being moved in the tube by means of a rack and pinion. A scale, divided into twentieths of a centim., is marked upon the brass portion of the rod, and a vernier is attached to the side of a section cut in the tube. Each of the ebonite rods carries at its end between the large plates a thin plane plate of glass; its surfuce facing the centre plate being covered with a thin metal foil. These two small plates are as nearly as pessible parallel to the parallel large plates, and their position between them is given by the vernier scale. The two outside plates, $\mathrm{B}$ and $\mathrm{C}$, are connected by a brass rod, as shown in fig. 1. At the centre of this rod is a brass ball kept well polished, which is capable of adjustment towards or away from the centre plate, by having the ends of the brass rod made to slide in tubes fitted to the two outside plates. The ball may also be rotated about the rod as an axis, or moved up and down. Another ball, nearly opposite the first, is fastened to the centre plate by a short metal pin, which permits it also to be rotated. This arrangement of the two balls allows them to have a wide range of adjustment in relation to each other, and permits different portions of their untarnished surfaces to be brought opposite.

This apparatus is arranged as follows:-The two outside plates are connected to the earth. One terminal of a large induction-coil is also connected to the earth, while the other is attached to the ball, $n$, which is fastened to the centre plate. When the balls are separated about 6 or 8 millim., sparks pass between them when the coil is put in operation.

We have here an oscillating electric system. The lines of force divide themselves between the two halves of the apparatus, reaching from the centre plate to the two outside plates. In the region of the small plates, the field may be considered as practically uniform. Hence, if these two small plates are at equal distances from the centre plate, which we consider for 
the present as midway between the outside plates, they are always at the same potential with respect to each other.

If a slab of dielectric be now placed upon the centre plate and beneath the movable plate, it will have two effects : 1st, to make the capacity upon its side of the centre plate greater than it is upon the other side; and, 2nd, to raise the potential at any point above it to a higher value than is the potential at a corresponding point upon the opposite side of the middle plate. Therefore, in order to keep the two small plates at an equal potential in reference to each other, they must be placed at unequal distances from the centre plate. The value of the specific inductive capacity of a substance is deduced in a manner presently to be shown, from the relative positions of these plates. Either one or two slabs of dielectric may be used, and several different dispositions may be given to the apparatus in a manner serving to prove the correctness of the determinations.

As the object of the experiment is to make a comparison of the values of the specific inductive capacity when obtained under slowly and rapidly oscillating fields, it becomes necessary, in obtaining the value under the latter condition, to completely separate out any slow changes in potential, which the centre plate may be subject to, from the rapid ehanges which take place when oscillation occurs, and which alone are to be made use of. It follows as a consequence of the large self-induction of the secondary of the induction-coil, that the potential of the plate to which the coil is attached rises slowly at first, until, finally, it becomes sufficiently high to break down the dielectric of the spark-gap; the rapid oseillations occurring only during the passage of the sparks. The following method was successfully employed to tell when the movable plates had the same potential in reference to each other, and to "weed" out the effects of the rapid oscillation to be used from any effects of slow changes in potential which might take place.

Two fine wires were led from the movable plates to the primary of a highly insulated transformer which was distant about $2 \frac{1}{2}$ feet from the apparatus (figs. 1 and 2). The secondary of the transformer had its terminals end in a sparkgap. This was made by approximating the points of two needles, fastened to the end of a small slab of paraffin held in a wooden clamp. One needle was made to slide in a fine glass tube, to allow the length of the spark-gap to be readily adjusted. It may be mentioned here that any device which has an appreciable capacity as compared with that of the movable plates cannot be employed to show when these plates have 
the same potential, if the spark-gap is used directly connected to the plates; and such is the case when the apparatus is used with slowly varying fields. When the plates are connected with the primary of the transformer, it is not a disadvantage to add capacity to the secondary. However, after trying a large number of devices such as a telephone, electrometer, electroscope, \&c., a spark-gap was found to be the simplest, most accurate, and in all respects the most convenient arrangement both with slowly changing and oscillating fields. One reason why the spark-gap best serves the purpose is because it requires at least 300 volts to make a spark pass, and consequently small and irregular alterations in potential are not shown. An electrometer-needle, on the other hand, keeps up a continual oscillation which is very confusing. A lens was adjusted in front of the spark-gap, and the room darkened when readings were taken. The transformer which was finally adopted, atter many trials of different forms, consisted of fine wire wound upon two glass tubes which slid over each other ; the wire upon the inner one forming the primary, that upon the outer one the secondary. The inside tube was $1 \frac{1}{2}$ inches in diameter and 7 inches long. This tube was given a thin coating of paraffin to insure high insulation; and in this was traced, with the aid of a lathe, a uniform spiral groove, there being in all 55 turns, 16 to the inch. No. 42 copper wire was wound in this groove, and the ends, soldered to terminal loops of somewhat larger wire, were secured at the ends of the tubes with drops of sealing-wax. The secondary, made in the same manner, consisted of 116 turns of wire of the same size, wound in a groove 35 turns to the inch. When all is in proper adjustment for oscillating fields, and the two small plates are at unequal potentials, bright sparks pass at the detector spark-gap, whenever rapid oscillations in the potential of the centre plate occur. If, however, the two balls ( $p$ and $n$, fig. 1, Pl. III.) are so far separated that no sparks can pass between them, the potential of the centre plate is then only varying at a slow rate and no sparks pass at the detector spark-gap, for the change of induction in the secondary is not sufficiently rapid to raise the potential to the 300 volts or more required to break down the dielectric. This point was carefully tested by working the coil-which had a sparking distance of 4 inches-to its full capacity and so placing the movable plates as to be always at the greatest difference of potential in reference to each other. Unless sparks passed between the balls, no sparks whatever passed at the detector spark-gap. But whenever sparks did pass betwren the balls, so that rapid oscillations were taking place, Phil. Mag. S. 5. Vol. 39. No, 236. Jan. 1895. 
bright sparks at the spark-gap always occurred. This was considered proof that whenever the transformer was employed, only the effects of the rapid oscillations were being observed.

If, now, the transformer is dispensed with, and the wires from the movable plates are connected directly to the sparkgap, and the two balls ( $p$ and $n$, fig. 1 ) are separated so that no sparks can pass between them, then evillently we have a slowly changing field, and the movable plates are, as before shown, at the same potential in reference to each other when the sparks go out at the detector spark-gap. The manner of taking the observations and the results obtained will be given after describing the mathematical theory of the method.

\section{Theory of the Method.}

The apparatus may be disposed in two distinct ways:-1st. Where one block of dielectric is used which is placed in the afparatus, as shown in fig. 1. 2nd. Where two equal slabs of dielectric are used and disposed, as shown in fig. 4.

\section{Case I.- - Where one Slab of Dielectric is used.}

Let $\mathrm{V}$ be the potential at any instant of the plate $A$, while the potentials of $\mathrm{B}$ and $\mathrm{C}$ are always kept at zero. Let the adjustable plates, $M$ and $N$, be so placed as to have partitions apon opposite sides of $A$ where the potential at any instant is $V_{1}$. Then for the side $A B$ of the double condenser,

$$
-\int_{\mathrm{V}}^{\mathrm{V}_{1}} d \mathrm{~V}=\frac{4 \pi \sigma^{\prime}}{\mathrm{K}} \int_{0}^{d_{1}} d x+4 \pi \sigma^{\prime} \int_{d_{1}}^{d_{1}+d_{4}} d x
$$

where $\mathrm{K}$ is the specific inductive capacity of the dielectric to be measured (K, fig. 1 ), and $d_{1}, d_{4}$ are the distances shown in fig. 1 , and $\sigma^{\prime}$ is the surface-density upon the upper side of plate A. This gives

$$
V-V_{1}=\frac{4 \pi \sigma^{\prime}}{\overline{\mathbf{K}}^{-}} d_{1}+4 \pi \sigma^{\prime} d_{k^{*}} . \quad . \quad . \quad .
$$

Likewise for the condenser $\mathrm{AC}$,

$$
\mathrm{V}-\mathrm{V}_{1}=4 \pi \sigma d_{2}, . . . \quad . \quad .
$$

where $\sigma$ is the surface-density upon the lower side of plate $A$.

Equating the second members of (1) and (2) we obtain

$$
\mathrm{K}=\frac{\sigma^{\prime} d_{1}}{\sigma d_{2}-\sigma^{\prime} d_{4}} \cdot \text {. . . . . . }
$$

Considering a unit area in the middle of plate $\mathrm{A}, \sigma^{\prime}=e^{\prime}$ 
for the upper side of the plate, and $\sigma=e$ for the under side, where $e^{\prime}$ and $e$ are the quantities of electricity upon unit area of the respective sides. Whence we have for the capacities $c^{\prime}$ and $c$ of unit areas of the two sides, $c^{\prime}=e^{\prime} V=\sigma^{\prime} V$ and $c=e \mathrm{~V}=\sigma \mathrm{V}$, and $\sigma^{\prime}=\frac{c^{\prime}}{\overline{\mathrm{V}}}$, and $\sigma=\frac{c}{\overline{\mathrm{V}}}$. Substitute these values for $\sigma^{\prime}$ and $\sigma$ in (3), and

$$
\mathrm{K}=\frac{e^{\prime} d_{1}}{c d_{2}-c^{\prime} d_{4}} \ldots . . . . . .
$$

Now, if the dielectric is as large as the plates, and these are not too far apart, the field may be taken as uniform over an area, $A$, equal to that of one of the small plates, $M$ or $N$.

Hence,

$$
c^{\prime}=\frac{\mathrm{A}}{4 \pi\left(\frac{d_{1}}{\mathrm{~K}}+d_{4}+d\right)}, \ldots . . .
$$

and

$$
c=\frac{\mathrm{A}}{4 \pi\left(d_{2}+d_{3}\right)} \ldots . \quad \text {. . . . }
$$

Substitute these values of $e^{\prime}$ and $c$ in (4) and

$$
\mathrm{K}=\frac{\frac{d_{1}}{\overline{d_{1}}+d_{4}+d}}{\frac{d_{2}}{d_{2}+d_{3}}-\frac{d_{4}}{\frac{d_{1}}{\bar{K}}+d_{4}+d}} \cdot . \quad . \quad .
$$

Reduced,

$$
\mathrm{K}=\frac{d_{1} d_{3}}{d d_{2}-d_{3} d_{4}} \ldots . . . .
$$

$d_{4}$ may be taken $=0$, and then

$$
\mathrm{K}=\frac{d_{1} d_{3}}{d d_{2}}
$$

We shall now determine the best thickness to give to the dielectric so that errors made in adjusting the plate $\mathrm{N}$ will produce the smallest error in deducing the value of $K$. The errors made in measuring $d$ and $d_{1}$ may be neglected in comparison with the error made in setting the plate $N$. Now, taking the case where $d_{4}=0$, and where each pair of lirge plates are the same distance apart,

$$
K=\frac{d_{1} d_{3}}{\left.d(\bar{D})-d_{3}\right)} \cdot \cdot \cdot \cdot \cdot \cdot
$$


84 Mr. E. F. Northrup on the Specific Inductive

Here $D$ is the total distance between either pair of large plates. The variation in the value obtained for $\mathrm{K}$ when $d_{3}$ varies from its true value is the error. Call the error $\mathbf{E}$. Then

from $(10)$

$$
\frac{\partial \mathrm{K}}{\partial d_{3}}=\mathrm{E}=\frac{\mathrm{D} d_{1}}{d\left(\overline{\mathrm{D}}-\bar{d}_{3}\right)^{2}}, \quad . \quad . \quad .
$$

$$
d=\frac{d_{3} d_{1}}{\mathrm{~K}\left(\mathrm{D}-d_{3}\right)} .
$$

Substitute this value of $d$ in (11), and we obtain

$$
\mathbf{E}=\frac{\mathrm{KD}}{d_{3}\left(\mathrm{D}-d_{3}\right)} \cdot \quad \cdot \quad \cdot \quad \cdot \quad \cdot
$$

The error is least when $\partial E=0$. Differentiating (12) we obtain

which, put $=0$, gives

$$
\partial \mathrm{E}=\frac{-\mathrm{KD}\left(\mathrm{K}-2 d_{3}\right) \partial d_{3}}{d_{3}^{2}\left(\mathrm{D}-d_{3}\right)^{2}}
$$

$$
d_{3}=\frac{\mathrm{D}}{2} . \quad . \quad . \quad \cdot .
$$

This says that a variation of $d_{3}$ from its true value produces the minimum error in the value deduced for $K$ when the plate $\mathrm{N}$ is halfway between the two large plates $A$ and $C$. To have the plate in this position the following relations must hold :-

and from (9)

$$
d_{3}=d_{2}=\frac{\mathrm{D}}{2}=\frac{d+d_{1}}{2}
$$

Whence we obtain

$$
\mathrm{K}=\frac{d_{1}}{d}
$$

$$
\begin{array}{ll}
\frac{\mathrm{D}}{2} & =\frac{d_{1}}{\mathrm{~K}}+d_{1} \\
\text { or } & d_{1}=\frac{\mathrm{K}}{1+\mathrm{K}} \mathrm{D} . \quad . \quad . \quad . \quad . \quad .
\end{array}
$$

This result, of course, can only be of value when $\mathrm{K}$ is approximately known. 
CASE II.-Where two equal Slals of Dielectric are used.

In this case plate A (fig. 4) is placed exactly midway between the plates $B$ and $C$. Two equal slabs of dielectric are used, one being placed upon $\mathrm{C}$, the other upon $\mathrm{A}$. The thickness of the blocks of dielectric and the distances between $\mathrm{BA}$ and CA are determined by considerations respecting the approximate value of $\mathrm{K}$ for the dielectric. If these distances are correctly taken, positions may be found for plates $\mathrm{N}$ and $M$ where they are always at the same potential, and where they are approximately in the centre of the spaces between the plates and the dielectric.

The plate $\mathbf{N}$ is supported on three small ebonite posts upon the slab D. Or, in the case of any other substance than glass, a hole may be bored through the dielectric, through which the ebonite rod which carries $\mathbf{N}$ may pass. The capacities of the two sides of the apparatus will now always remain equal, for if the field alternates at different rates, both dielectrics will change their specific inductive capacities equally. The formula giving the value of $K$ is then obtained as follows :-

For the side $\mathrm{AB}$,

whence

$$
-\int_{\mathrm{V}}^{\mathrm{V}_{1}} d \mathrm{~V}=\frac{4 \pi \sigma}{\mathrm{K}} \int_{0}^{d_{1}} d x+4 \pi \sigma \int_{d_{1}}^{d_{1}+d_{4}} d x ;
$$

$$
\mathrm{V}-\mathrm{V}_{1}=\frac{4 \pi \sigma}{\mathrm{K}} d_{1}+4 \pi \sigma d_{4} . . . .
$$

For the side $A C$,

$$
\mathrm{V}-\mathrm{V}_{1}=4 \pi \sigma d_{2} \ldots \text {. . . . . }
$$

Equate (15) and (16), and we obtain

$$
\mathrm{K}=\frac{d_{1}}{d_{2}-d_{4}} . \quad . \quad . \quad . \quad . .
$$

Use of Apparatus according to First Method.

According to the theory given under Case I., the value of $\mathrm{K}$ may be obtained when the capacities of the two sides of the apparatus are unlike and unknown. It was found, however, unless the apparatus is so disposed that the capacities of the two sides are nearly equal, that the value of $K$ obtained is not quite exact in the case of slowly varying fields, and that with oscillating fields no result whatever can be obtained. The formula is based upon the assumption that static conditions hold. Now the formula assumes that, when the centre plate has a given potential, the surface-density of each side of 
the plate is proportional to the capacity of the corresponding side of the apparatus. This, however, is not strictly true. As the alternations grow more rapid, the average surface-densities upon the two sides of the middle plate tend to become equal, though the capacities of the two sides of the apparatus are unlike. Moreover, if the capacities of the two sides differ largely, there is such an interference of waves, when rapid oscillations are taking place, that no position can be found for the movable plates where their potentials in reference to each other will always be equal, and consequently the sparks in the detector spark-gap cannot be made to disappear. This diffculty is not observable if the capacities are made nearly the same. It becomes necessary then, in using the apparatus with one slab of dielectric, to have the capacities of the two sides approximately equal. This adjustment can be made in a manner to be shown presently.

In taking observations by this method we proceed thus : The plates $M$ and $N$ are first brought into contact with the centre plate and the zero positions upon the scales are determined. The block of dielectric to be tested is then placed upon the plate $A$, and plate $M$ is brought to rest upon it. The reading upon the vernier scale gives the thickness of the block. Great care, of course, is taken to cut this block so that its two faces are parallel. We may now leave plate M resting upon the dielectric or give it any desired position above it. The wires leading from $\mathrm{N}$ and $\mathrm{M}$ are first connected directly to the spark-gap and the balls, $p$ and $n$, are so far separated that no spark can pass between them. In the experiment as tried, two large coils were connected in series so as to make the period of the slowly changing field as long as possible. The coil being set in action, the sparks at the detector spark-gap are observed, and the plate $\mathrm{N}$ is slowly moved up and down until a point is found where the sparks entirely disappear. There is a distance of about 2 millim. through which the plate may be moved without the sparks appearing. A reading is taken at the upper and at the lower limit of this space, and the mean taken as the true reading. The limits of accuracy of the method are confined to the accuracy with which this setting of the plate $\mathrm{N}$ can be made. Any other error should be attributed to crudeness in the construction of the apparatus, and not be considered as intrinsic to the method. These readings so taken, give by applying formula (8) an approximate value of $\mathbf{K}$ for slowly changing fields. The capacities of the two sides may now be made nearly equal by approaching the two lower plates until they are the proper distance apart. This distance is determined 
as follows:--Using the nearly correct value of $\mathrm{K}$ just obtained, we have for the capacity of the side $\mathrm{AB}$, taking $d_{4}=0$,

$$
\mathrm{C}_{1}=\frac{\mathrm{A}}{4 \pi\left(\frac{d_{1}}{\mathrm{~K}}+d\right)} . . . .
$$

For the side $\mathrm{AC}$,

$$
\mathrm{C}=\frac{\mathrm{A}}{4 \pi \mathrm{D}} . \quad . \quad . \quad . \quad .
$$

Fquating the right-hand members of (18) and (19), we obtain

$$
\mathrm{D}=\frac{d_{1}+\mathrm{K} d}{\mathrm{~K}}, . . . .
$$

This value of $\mathrm{D}$, then, makes the capacities of the two sides the same, within the limits in which $\mathrm{K}$ is known. A still closer value of $\mathrm{K}$ may now be obtained as before. Two adjustments of the apparatus are sufficient for an accurate determination. If the adjustment is exactly right $\sigma$ and $\sigma^{\prime}$ cancel out in expression (3), and hence

$$
\mathrm{K}=\frac{d_{1}}{d_{2}-d_{4}} .
$$

Readings should now be taken with the different values given to $d_{4}$. The closeness of the agreement of the values of $K$ then obtained furnish a test of their accuracy.

To obtain, next, the value of $\mathrm{K}$ under oscillating fields we proceed as follows:- The two balls, $n$ and $p$, are approached so that sparks can pass between them, the distance being chosen such that the best conditions may exist for oscillations taking place. The movable plates are then comnected to the primary of the transformer, and the spark-gap to the secondary. Great care should be taken thit the primary leads are symmetrically arranged and of the same length, and that no conductor be near them. Carelessness on this point led for some time to conflicting results. Waves of energy should reach the transformer along both leads at the same instant, which they will not do if the leads are of unequal length. The plates are set by means of the spark-gap, as in the case of slowly changing fields. If the value of $\mathrm{K}$ comes out much smaller than in the case of slow fields, another adjustment of the apparatus may be necessary. This being made, another determination can be made for slowly varying fields, using formula (8), which for small differences in the capacities of the two sides gives results correct within the limits of other necessary errors. Thus, after the apparatus has been correctly arranged, a comparison by this 
method of the values of $\mathrm{K}$ under slowly changing and oscillating fields is possible, without changing the plates. However, if the capacities are equal for the two sides when slowly changing fields are used, it was found by a careful test that the simple formula, $\mathrm{K}=\frac{d_{1}}{d_{2}-d_{4}}$, may still be used when the field is oscillating, although the capacities of the two sides are now slightly different. The reason for this is that with oscillating fields the average surface-densities upon the two sides of the centre plate are the same, and hence cancel out of formula (3). The proof of this rests upon the fact that $K$ was found to be the same for all positions of $d_{4}$, and this would not be the case if the formula were incorrect. Thus, as shown,

Let $\sigma=\sigma^{\prime} \pm \Delta \sigma^{\prime}$, then

$$
\mathrm{K}=\frac{d_{1} \sigma^{\prime}}{\sigma d_{2}-\sigma^{\prime} d_{4}}
$$

$$
\mathrm{K}=\frac{d_{1} \sigma^{\prime}}{d_{2}\left(\sigma^{\prime} \pm \Delta \sigma^{\prime}\right)-\sigma^{\prime} d_{4}}=\frac{d_{1}}{d_{2}-d_{4} \pm \frac{\Delta \sigma^{\prime}}{\sigma^{\prime}} d_{2}} . .
$$

Now whenever $d_{4}$ is changed $d_{2}$ must change. Hence if $\Delta \sigma^{\prime}$ has any value, $\mathrm{K}$ must change with $d_{4}$ if calculated by the formula $K=\frac{d_{1}}{d_{2}-d_{4}}$, but as a series of tests showed, using the simple formula to calculate the value of $K$, that its value did not change, $\Delta \sigma^{\prime}=0, \sigma=\sigma^{\prime}$; and hence under the conditions stated, the simple formula, $K=\frac{d_{1}}{d_{2}-d_{4}}$, may be used.

Use of the Apparatus according to the Second Method.

The disposition of the apparatus discussed under Case II. admits of results being obtained more quickly and satisfactorily than in the one just described, and should be preferred to the other if two equal slabs of dielectric are obtainable. Readings are taken in the same manner as with the other disposition of the apparatus. The results may be tested in a variety of ways which are readily suggested by the above discussion.

\section{Observations upon Paraffin.}

The first substance experimented upon was hard paraffinwax. The block used was a little larger than the plates and 4.34 centim. thick. Its melting-point was determined to be $542_{2}^{\circ}$ Centigrade. Only one suitable block was readily obtainable, and so the first method was employed. Previously 
to obtaining the final result many different dispositions of the apparatus were tried, and the method tested in most of its features. Experiments were also made upon a block of paraffin smaller than the large plate; but it was found that the results were incorrect in this case, which fact makes it necessary to use blocks of the same size as or larger than the plates of the apparatus.

In obtaining the final values, the apparatus was adjusted so that $d_{2}+d_{4}=3.51$ centim., and $\mathrm{D}$ was changed until the capacities of the two sides were nearly equal. In all cases superior and inferior readings were taken as previously described, and the mean taken as the true reading. The following characteristic set of ten readings, taken with slow fields, are given to illustrate the accuracy with which the plate may be adjusted. One scale-division $=\frac{1}{2}$ centim.

$$
\begin{aligned}
& d_{4}=2 \text { scale-divisions. } \\
& \begin{array}{l|l}
1 & 13 \cdot 82
\end{array} \\
& \begin{array}{l|l}
2 & 14 \cdot 32
\end{array} \\
& 3 \quad 13 \cdot 80 \\
& 4 \quad 14 \cdot 20 \\
& 5 \quad 13 \cdot 78 \\
& 6 \quad 14 \cdot 22 \\
& \begin{array}{lll}
7 & 13 \cdot 80
\end{array} \\
& 8 \quad 14 \cdot 22 \\
& 9 \quad 13 \cdot 79 \\
& 10 \quad 14 \cdot 20 \\
& 14 \cdot 015=\text { mean. } \\
& 8 \cdot 450=\text { zero-reading of scale. } \\
& 5 \cdot 565=d_{2} \text {. }
\end{aligned}
$$

The final values obtained for paraffin are embodied in the following table. The value for slow fields differs a trifle from that published in a preliminary article; further experience with the apparatus having led to the present value.

\section{Slow Fields.}

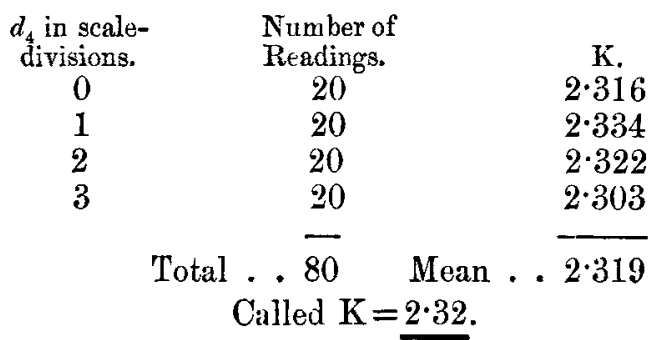




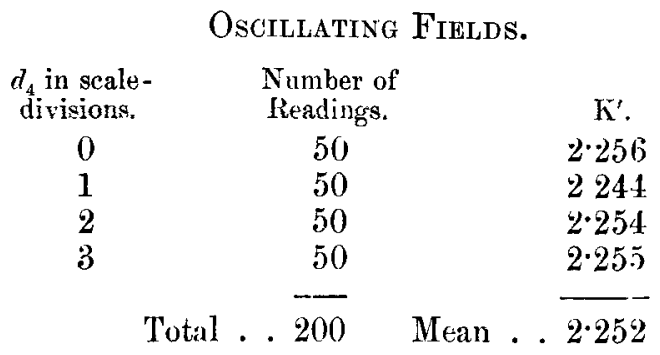

Called $\mathrm{K}^{\prime}=2 \cdot 25$.

Thus $\mathrm{K}$ for praraffin is 3 per cent. less in oscillating fields than in slowly varying fields.

\section{Olsertations upon Gluss.}

Plate-glass seems to be the most important substance upon which to experiment, as its specific inductive capacity has been found very different by different observers, and because the values of $K$ found for it are much greater than the square of its index of refraction. The greatest pains were taken to obtain certain results for this substance; and as the values obtained differ considerably from those found by other observers, it is hoped my readers will pardon the mention of some tedious details.

The second method was employed as described under Case II. Twelve plates of American plate-glass were used, six plates being placed in each half of the apparatus. By making the proper selection of plates for each side, the two piles were made of the same thickness. The density of this glass was determined with a Jolly balance and found to be $2 \cdot 678$. All measurements of dimensions were made with an excellent pair of vernier calipers and repeated a large number of times. Referring to fig. 4 for the meaning of the letters, the following dimensions were employed. These are all giren in scale-divisions which equal one half the metric system :$\mathrm{P}=10 \cdot 21, d_{1}=11 \cdot 964, d_{2}=5 \cdot 010, d_{4}=\chi$.

In order that there might be no springing of the plates after all had been adjusted, eight ebonite posts were placed at the corners as shown in fig. 4. The plate $\mathrm{N}$ was supported upon three thin tubes of ebonite. All surfaces where there could be any harmful leakage of electricity, such as along the supports to the plates $\mathrm{M}$ and $\mathrm{N}$, were covered with a thin coating of paraffin. The small plates were connected to the spark-gap or transformer with two No. 36 bare copper wires, 
each $2 \frac{1}{2}$ feet long. These wires were supported near the spark-gap at the ends of glass rods covered with paraffin. The transformer and spark-gap were also carefully insulated by glass and paraffin. These precautions are necessary by reason of the fact that a small leakage of electricity from the surface of the small plates changes the reading in a marked manner. Leakage of electricity, however, could be seen by darkening the room, and could also be detected by a peculiar manner in which the sparks went out. This was carefully looked for and avoided. In making the test with oscillating fields, half of the readings were taken with the transformer in one position and then the other half were taken with the ends of the transformer reversed, and the mean of the two series taken as the true reading. This becomes necessary unless the primary and secondary of the transformer are symmetrically arranged in reference to each other. Indeed it was found that the readings could be perceptibly changed by sliding the secondary coil over the primary into different positions. It is easily seen, however, that if the primary and secondary always have the same relation, and the connexions to the primary are reversed after half the readings are taken, the error is eliminated in the mean of all the readings. The current through the coil was at times large and again small, and also occasionally reversed ; but these changes, as expected, did not affect the results. The length of the indicator spark-gap could also be changed without affecting the mean reading. Rearlings for slow fields were taken in sets of ten and averaged. The mean of twenty such sets, taken with slow fields, was $3 \cdot 097$. The greatest departure from the mean was $+\cdot 09$. Finally, from $(17)$, when $d_{1}=11 \cdot 961$, $d_{2}=5 \cdot 010, d_{4}=3 \cdot 097$, we obtain $\mathrm{K}=6 \cdot 254$.

$$
\text { Called } \mathrm{K}=\underline{6 \cdot 25} \text {. }
$$

The value was next obtained for oscillating fields, no change being made in the apparatus, exeept to connect in the transformer and approach the balls to the proper distance for sparks to pass and cause oscillations. Readings were taken in sets of twenty each. Following is the average of each of the sets taken :- 


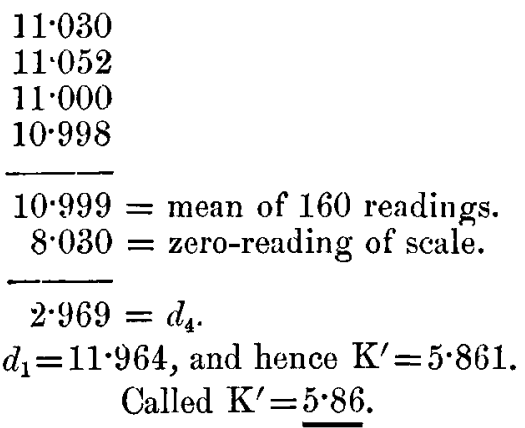

These results, then, give the specific inductive capacity of plate-glass 6.2 per cent. less in oscillating than in slowly changing fieids.

Though the relative values of $\mathrm{K}$ for glass under these two conditions seemed to be undoubtedly correct, it was not expected to find such a low value for slow fields; and so, to verify the results obtained, the apparatus was taken apart and put together again with the dimensions changed and the experiment repeated for slow fields. The distances chosen were $\mathrm{P}=6.401, d_{2}=3.524, d_{1}=11.964$; and as the result of thirty readings $d_{4}=1.574$. Whence $K=6.14$, or 1.8 per cent. less than before.

These results show that there is a decrease in the value of $\mathrm{K}$ for paraffin and glass when the alternations of force are rapid, but that the change is much smaller than several observers have stated. M. Blondlot found $\mathrm{K}$ for glass in oscillating fields to be $2 \cdot 84$. J. J. Thomson obtained for glass the value $2 \cdot 7$ (see 'Recent Researches in Electricity and Magnetism,' by J. J. Thomson, p. 471 and following). Ewing found the value of mirror-glass by a method of oscillations (Physical Review, July and August 1894, p. 51) to be 5.84. His calculated value for glass was $6 \cdot 24$.

The above method, it is believed, is capable of giving accurate results if the apparatus be well made, and it is hoped that the detailed description given above will enable anyone to readily repeat the experiment. Should this be done, 1 would suggest that the apparatus might with advantage be made considerably smaller.

I desire to say, in conclusion, that I am greatly indebted to Professor Henry A. Rowland for suggestions, and especially for the opportunity of performing the experiment*.

* The entire original idea of this method and all its details are due to Mr. Northrup, and only one or two very minor points are my own.Henry A. Rowland. 